\title{
A rare vascular anomaly in the form of double left brachiocephalic vein detected incidentally during cardiac implantable electronic device (CIED) placement
}

\author{
R. Steckiewicz, E.B. Świętoń, P. Stolarz \\ Department of Cardiology, Medical University of Warsaw, Poland
}

[Received: 4 June 2017; Accepted: 21 June 2017]

\begin{abstract}
The growing number of transvenous cardiac implantable electronic device (CIED) implantation procedures helps detect rare vascular anomalies. Genetic disturbances in vascular development can produce systemic vein anomalies, including the left brachiocephalic vein (BCV). BCV anomalies commonly coexist with a persistent left superior vena cava (PLSVC), detected in $0.3-0.5 \%$ of the general population. The three known anatomical variations of PLSVC are two variations involving a $B C V$ bridge and the third with BCV agenesis. BCV anomalies occur in $1 \%$ of patients with congenital heart defects, whereas the estimated proportion of $B C V$ anomalies in the population with no cardiovascular symptoms is below $0.4 \%$. A rarely observed, and thus rarely reported, $B C V$ variation is a double left $B C V$, with the additional vessel typically found inferior and posterior to the ascending aorta prior to draining into the superior vena cava. This case report presents a previously unreported variation of double left $B C V$, with both vessels coursing parallel to each other, superior to the aortic arch. (Folia Morphol 2018; 77, 1: 161-165)
\end{abstract}

Key words: double left brachiocephalic vein, venography, computed tomography, cardiac pacing

\section{INTRODUCTION}

The growing number of invasive transvenous procedures, including cardiac implantable electronic device (CIED) placement, facilitates detection of rare vascular anomalies. The, initially symmetrical, venous system in normally developing human embryos is composed of two pairs of cardinal veins, with the anterior cardinal veins draining the cranial part of the embryo. At the eighth week of gestation, the paired anterior cardinal veins anastomose to form the brachiocephalic vein $(B C V)$. Any disruption of this stage of embryonic development may result in BCV anomalies, including a double left $B C V$, presented in this paper $[3,10,11,14,17]$.

The left BCV is formed by the confluence of the left internal jugular vein (IJV) and the left subclavian vein and is their anatomical continuation. The left $\mathrm{BCV}$ is typically located superior to the aortic arch and anterior to its main branches: the brachiocephalic trunk, the left common carotid artery, and the left subclavian artery. The left BCV enters the right superior mediastinum and joins the right $\mathrm{BCV}$ to form the superior vena cava (SVC) (Fig. 1). 


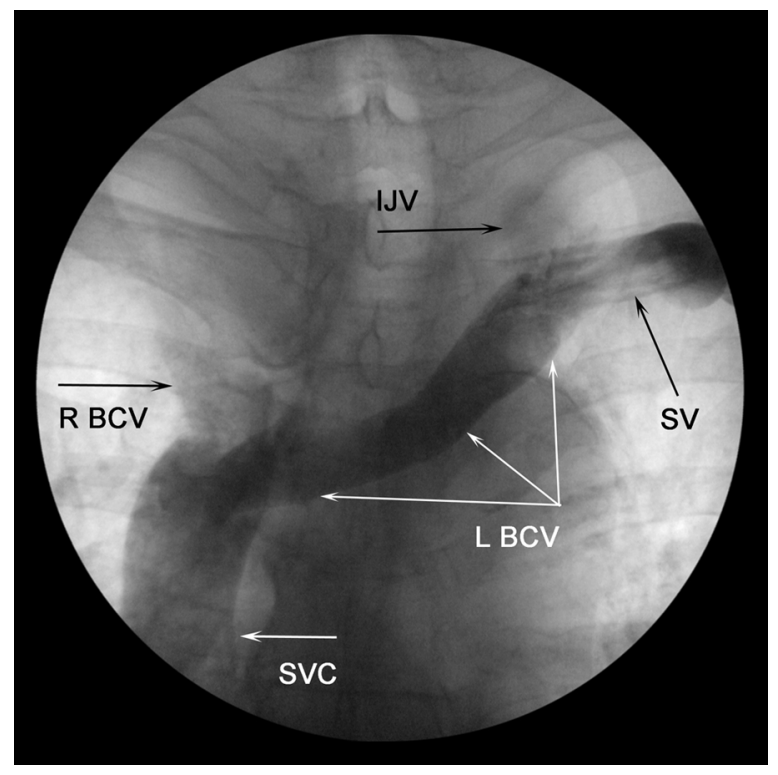

Figure 1. Superior mediastinal vein topography that facilitates lead insertion during transvenous cardiac implantable electronic device (CIED) procedures (A-P view, with contrast); BCV — brachiocephalic vein; L — left; R — right; SV — subclavian vein; SVC superior vena cava; IJV — internal jogular vein.

One of the consequences of developmental venous anomalies is the presence of a persistent left superior vena cava (PLSVC), detected in $0.3-0.5 \%$ of the general population, and occurring in three anatomical variations, differing in terms of the presence and course of the BCV $[4,9,15,16]$.

In up to $1 \%$ of cases BCV anomalies are associated with congenital heart defects, such as tetralogy of Fallot, patent foramen ovale, or ventricular septal defect. The prevalence rates reported in the literature depend on the evaluated population and imaging techniques employed. In the population with no heart defects, the estimated prevalence of this venous anomaly is under $0.4 \%[1,2,7,12]$.

One of the infrequently occurring and reported $B C V$ anomalies is the presence of a double left BCV $[8,11,18]$. In this form of the anomaly, the additional, left BCV typically courses posterior and inferior to the ascending aorta prior to joining the right BCV.

Here, we presented a variation of the double left $B C V$, with the upper and lower vessel running parallel to each other and superior to the aortic arch. This anomaly was detected incidentally during cardiac pacemaker implantation. The authors found no similar venous configuration reported in the available literature.

\section{CASE REPORT}

An 82-year-old male was qualified for permanent cardiac pacing due to a history of tachycardia-bradycardia syndrome with episodes of cardiovascular presyncope (sinus bradycardia, paroxysmal atrial fibrillation). On February 27, 2017, under local anaesthesia administered to the left pectoral region, the cephalic vein was dissected in the clavipectoral triangle. Following cephalic vein cutdown, we, unsuccessfully, attempted to advance a lead through the venous system (Fig. 2A, B). In order to determine the reason behind the encountered difficulty, venography was performed, with the contrast medium administered through the cutdown incision (Fig. 2A, C).

A contrast agent, administered to visualise full topography and morphometry of the venous system in the superior mediastinum, revealed the presence of two patent left BCVs.

Both vessels coursed above the aortic arch and anteriorly to its arterial branches. The lower vessel of the left BCV drained to the already formed SVC somewhat below the confluence of the upper left BCV with the right $\mathrm{BCV}$. One characteristic morphological feature of the lower vessel of the left BCV was a vertical course of its initial segment and its nearly right-angle bend at the level of the aortic arch. This shape of the lower vessel of the left BCV prevented lead advancement (Fig. 2B). Eventually, a DDD-mode Sensia DR pacemaker (Medtronic) was implanted subcutaneously in the left pectoral region, with both leads (an atrial lead, model 5076-52, and a ventricular lead, model 5076-58) inserted via subclavian vein puncture and advanced through the upper vessel of the double left BCV.

Postoperative computed tomography angiography of veins in the superior mediastinum showed detailed topography and morphometry of the double left BCV, which helped determine its spatial correlation with neighbouring anatomical structures (Fig. 3B, C).

\section{DISCUSSION}

Normal formation of the venous system during embryonic development produces the left BCV coursing obliquely from the left to the right in the superior mediastinum, superoanteriorly to the aortic arch to join the right BCV and form the right SVC.

One of the rare developmental abnormalities of the BCV is associated with another developmental venous anomaly in the form of the PLSVC. This anomaly occurs in three anatomical variations. In the case of 


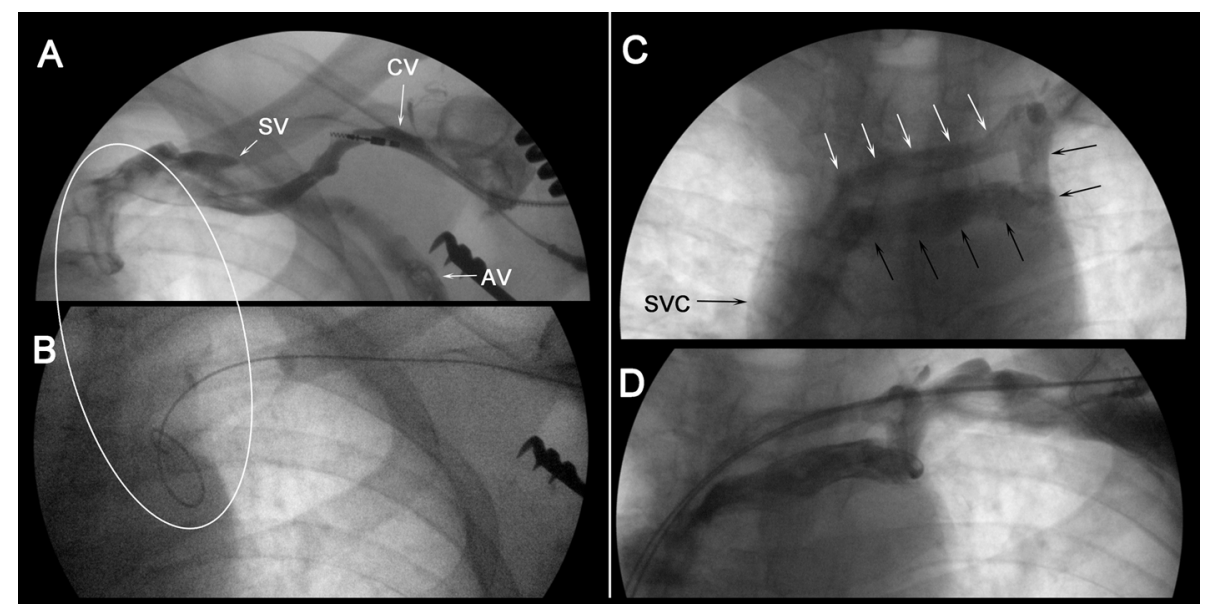

Figure 2. A-D. Stages of the cardiac implantable electronic device implantation procedure described in this paper. A. A venogram illustrating the site of difficulties in lead advancement through the cephalic vein (CV), and the anatomical dissimilarity of this vascular segment (upper part of the oval) in comparison to its typical topography (Fig. 1); SV — subclavian vein; AV — axillary vein; B. Fluoroscopy. The site of unsuccessful guidewire advancement (lower part of the oval) in reference to the vessel in this location (A). C. Re-venography revealed the presence of double left brachiocephalic vein (BCV): the upper vessel (white arrows) and the lower, wider, vessel (black arrows) with a right-angle bend in its initial segment (circle in panels $A, B$ ). D. Eventually, both guidewires and leads were advanced to the superior vena cava (SVC) through the upper vessel of the left BCV.

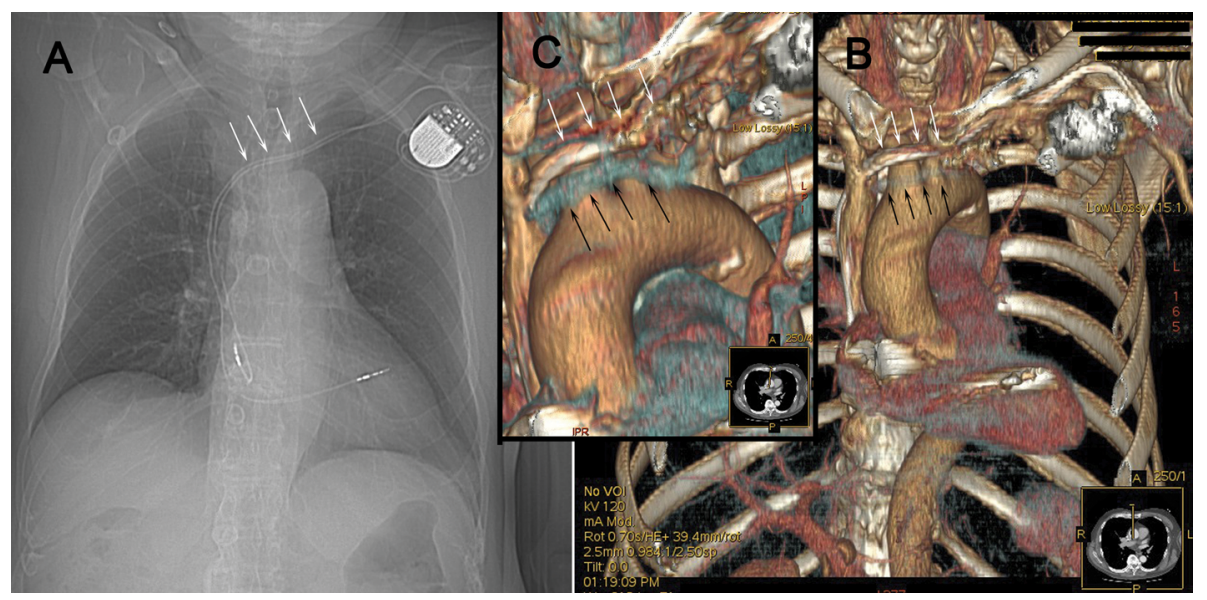

Figure 3. A-C. Computed tomography (CT) angiography. A. A-P view of the chest shows vein topography, indicated by the presence of the leads coursing within. B. CT reconstruction adjusted to illustrate the upper vessel of the double left brachiocephalic vein (BCV) after its confluence with the right $\mathrm{BCV}$ to form the superior vena cava. Cardiac leads (white arrows) can be seen within the vascular lumen. C. CT reconstruction adjusted to illustrate the lower vessel of the double left BCV, measuring $8 \mathrm{~mm}$ in diameter (black arrows).

bilateral SVC both vessels are connected by a "venous bridge" and form the right and left BCVs. In rare cases of isolated PLSVC the "venous bridge" formed by both BCVs is connected only to the PLSVC due to agenesis of the right SVC. An equally rare variation is the presence of bilateral SVC with concomitant agenesis of both BCVs $[9,15,16]$.

Another rarely detected and reported type of left $\mathrm{BCV}$ anomaly is the presence of an additional left BCV. In cases when such an extra vessel forms, it typically courses lateral to the aortic arch and passes under- neath it to eventually join the right BCV posterior to the ascending aorta $[1,5,12]$.

We believe the rates of left $\mathrm{BCV}$ variations are higher than suggested by the number of reported cases. These venous anomalies escape detection during invasive procedures when cardiac leads can be successfully advanced through venous lumina and there is no need for intraoperative imaging. For example, if the anatomical layout of the double left BCV draining into the SVC is favourable, a chance insertion of the guidewire into the lower vessel of the double left BCV is possible (Fig. 4A, B). 

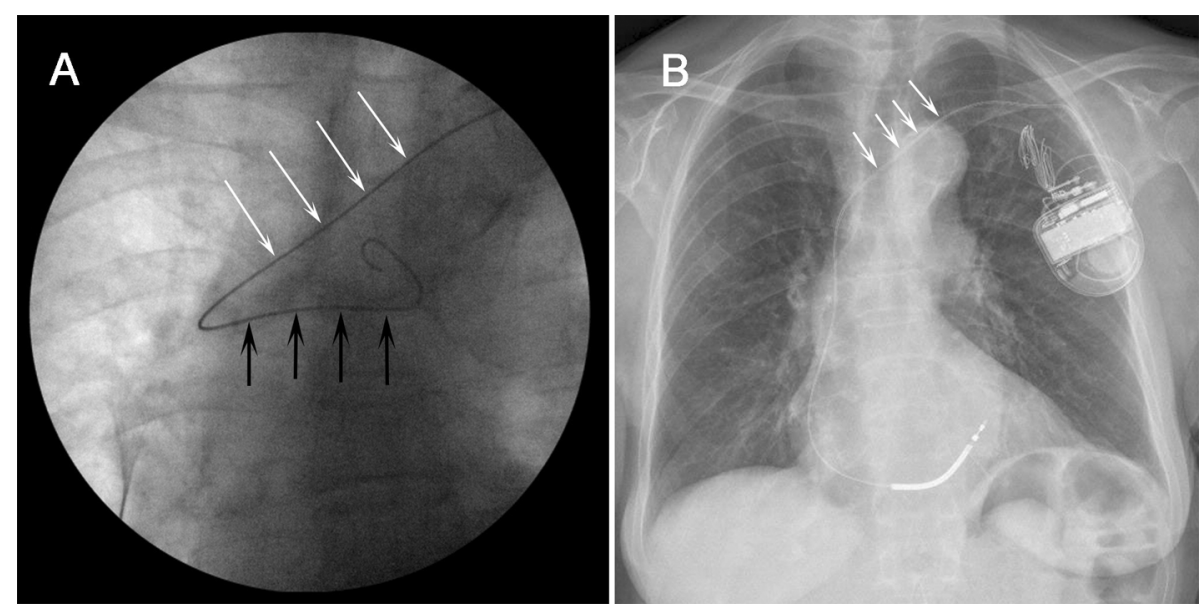

Figure 4. A, B. A 78-year-old woman with an implantable cardioverter-defibrillator (Maximo II VR, Medtronic), with a model 6935-65 lead, implanted in 2013. A. Fluoroscopy: the shape of the intravenously inserted guidewire indicates the presence of a double brachiocephalic vein $(\mathrm{BCV})$. The oblique position of the guidewire in the superior mediastinum, coursing from the upper left subclavian vein to the lower right (superior vena cava), illustrates the typical location of the left BCV (white arrows), whereas the guidewire segment coursing practically horizontally from the right to the left suggests the presence of an additional, infra-aortic form of the left BCV (black arrows). Venography was not performed due to the patient's allergy to contrast media. B. Chest X-ray. illustrating the probable location of the additional form of the left BCV (oval) with respect to the aortic arch and tracheal bifurcation.

De-novo CIED implantation typically involves the use of veins coursing in the left clavipectoral triangle. Some centres perform de-novo CIED implantation procedures on the right side (whether on the patient's request or due to past injury to the left pectoral region or for other reasons). Such situations limit the rate of double left BCV detection, which precludes accurate estimation of the rates of this anomaly.

There is a case report describing the co-existence of both anomalies (PLSVC and double left BCV), with the additional vessel of the double left BCV branching off the PLSVC [13]. This is similar to the case reported by Kawamura et al. [8], where - as in the case presented here - the shape of the initial segment of the lower vessel of the left $B C V$ resembled a partly formed and haemodynamically patent PLSCV (Fig. 2C, D). This aetiology may be supported by reports that include the morphometry and topography of fully developed PLSVC [16]. In the case presented here, the remaining part of the PLSVC may have undergone physiological atrophy in the embryonic period.

Despite being clinically asymptomatic, a double left BCV, if present, may significantly affect the course of various invasive procedures, just as it did in the case presented here. The rates of detecting venous anomalies during CIED-related procedures will increase along with the growing number of these procedures. This is due to a growing variety of CIEDs, with devices offering tachyarrhythmia management and cardiac resynchronisation therapy devices becoming more and more common [6].

\section{Limitations of the study}

The theory as to the developmental precursor of the initial segment of the lower vessel of the double left BCV may seem dubious; nonetheless, considering the morphometry and topography of PLSVC cases reported in the literature, it is - in fact - highly probable.

\section{CONCLUSIONS}

This case report is the first one, to the authors' knowledge, describing a double left BCV with this topography revealed during pacemaker implantation.

\section{REFERENCES}

1. Chen SJ, Liu KL, Chen HY, et al. Anomalous brachiocephalic vein: $\mathrm{CT}$, embryology, and clinical implications. AJR Am J Roentgenol. 2005; 184(4): 1235-1240, doi: 10.2214/ ajr.184.4.01841235, indexed in Pubmed: 15788602.

2. Chern MS, Ko JS, Tsai A, et al. Aberrant left brachiocephalic vein: $C T$ imaging findings and embryologic correlation. Eur Radiol. 1999; 9(9): 1835-1839, indexed in Pubmed: 10602959.

3. Corno AF, Alahdal SA, Das KM. Systemic venous anomalies in the Middle East. Front Pediatr. 2013; 1: 1, doi: 10.3389/ fped.2013.00001, indexed in Pubmed: 24400249. 
4. Ghadiali N, Teo LM, Sheah K. Bedside confirmation of a persistent left superior vena cava based on aberrantly positioned central venous catheter on chest radiograph. Br J Anaesth. 2006; 96(1): 53-56, doi: 10.1093/bja/aei272, indexed in Pubmed: 16311276.

5. Gülsün M, Gökoğlu A, Ariyürek M, et al. Subaortic left brachiocephalic vein: computed tomography and magnetic resonance angiography findings. Surg Radiol Anat. 2003; 25(3-4): 335-338, doi: 10.1007/s00276-003-01203, indexed in Pubmed: 12910378.

6. Hindricks G, Camm J, Merkely B, et al. The EHRA White Book. 2016: 375-384.

7. Kahkouee S, Sadr M, Pedarzadeh E, et al. Anomalous left brachiocephalic vein: important vascular anomaly concomitant with congenital anomalies and heart diseases. Folia Morphol. 2017; 76(1): 51-57, doi: 10.5603/ FM.a2016.0031, indexed in Pubmed: 27830886.

8. Kawamura I, Hojo R, Fukamizu S. A case of pacemaker implantation in the patient with duplication of the left innominate vein: a case report. Springerplus. 2016; 5: 515, doi: 10.1186/s40064-016-2182-9, indexed in Pubmed: 27186479 .

9. Kawashima T, Sato K, Sato F, et al. An anatomical study of the human cardiac veins with special reference to the drainage of the great cardiac vein. Ann Anat. 2003; 185(6): 535-542, doi: 10.1016/50940-9602(03)80122-X, indexed in Pubmed: 14703998.

10. Ko SF, Huang CC, Ng SH, et al. Imaging of the brachiocephalic vein. AJR Am J Roentgenol. 2008; 191(3): 897-907, doi: 10.2214/AJR.07.3552, indexed in Pubmed: 18716126.

11. Kondrachuk O, Yalynska T, Tammo R. Double left brachiocephalic vein. Pediatr Cardiol. 2013; 34(3): 767-768, doi: 10.1007/s00246-012-0542-y, indexed in Pubmed: 23052674
12. Kulkarni S, Jain S, Kasar P, et al. Retroaortic left innominate vein - Incidence, association with congenital heart defects, embryology, and clinical significance. Ann Pediatr Cardiol. 2008; 1(2): 139-141, doi: 10.4103/0974-2069.43881, indexed in Pubmed: 20300257.

13. Kwon O, Lim J, Lee J, et al. Double left brachiocephalic veins with persistent left superior vena cava: a case report. J Korean Society Radiol. 2014; 71(2): 55, doi: 10.3348/ jksr.2014.71.2.55.

14. Nagashima M, Shikata F, Okamura T, et al. Anomalous subaortic left brachiocephalic vein in surgical cases and literature review. Clin Anat. 2010; 23(8): 950-955, doi: 10.1002/ca.21046, indexed in Pubmed: 20830788.

15. Povoski SP, Khabiri H. Persistent left superior vena cava: review of the literature, clinical implications, and relevance of alterations in thoracic central venous anatomy as pertaining to the general principles of central venous access device placement and venography in cancer patients. World J Surg Oncol. 2011; 9: 173, doi: 10.1186/14777819-9-173, indexed in Pubmed: 22204758.

16. Steckiewicz R, Kosior DA, Rosiak M, et al. The prevalence of superior vena cava anomalies as detected in cardiac implantable electronic device recipients at a tertiary cardiology centre over a 12-year period. Hellenic J Cardiol. 2016; 57(2): 101-106, doi: 10.1016/j.hjc.2016.03.003, indexed in Pubmed: 27445024.

17. Takada Y, Narimatsu A, Kohno A, et al. Anomalous left brachiocephalic vein: CT findings. J Comput Assist Tomogr. 1992; 16(6): 893-896, indexed in Pubmed: 1430437.

18. Topcuoglu OM, Atceken Z, Ariyurek OM. Circumaortic doubled left brachiocephalic vein: a rare confusing variation. Surg Radiol Anat. 2015; 37(3): 315-318, doi: 10.1007/ s00276-014-1345-z, indexed in Pubmed: 25037739. 